Article

\title{
Spectral-Spatial Dimensionality Reduction of APEX Hyperspectral Imagery for Tree Species Classification; a Case Study of Salzach Riparian Mixed Forest
}

\author{
Zahra Dabiri * and Stefan Lang \\ Department of Geoinformatics (Z_GIS), Salzburg University, Schillerstr.30, 5020 Salzburg, Austria; \\ (firstname).(secodname)@sbg.ac.at \\ * Correspondence: Zahra.dabiri@sbg.ac.at; Tel.: +43-662-8044-7562
}

\begin{abstract}
Tree species composition is an important key element for biodiversity and sustainable forest management, and hyperspectral data provide detailed spectral information, which can be used for tree species classification. There are two main challenges for using hyperspectral imagery: a) Hughes phenomena, meaning by increasing the number of bands in hyperspectral imagery, the number of required classification samples would increase exponentially, and b) in a more complex environment, such as riparian mixed forest, focusing on spectral variability per pixel may not be adequate for definability of tree species. Therefore, the focus of this study is to assess spectral-spatial dimensionality reduction of airborne hyperspectral imagery by using minim noise fraction (MNF) transformation, and object-based image analysis (OBIA). An airborne prism experiment (APEX) hyperspectral imagery was used. A study area was a riparian mixed forest located along the Salzach river, and six tree species including Picea abies, Populus (canadensis and balsamifera), Fraxinus excelsior, Alnus incana, and Salix alba were selected. A machine learning algorithm random forest (RF) was used to train and apply a prediction model for classification. Using a spectral dimensionality reduced APEX, a pixel-level classification was also done. According to a confusion matrix, the object-level classification of MNF-derived components achieved the overall accuracy of $85 \%$, and kappa coefficient of 0.805 . The performance of classes according to producer's accuracy varied between $80 \%$ for Fraxinus excelsior, Alnus incana, and Populus canadensis to $90 \%$ for Salix alba and Picea abies. Comparison the results to a pixel-level classification, showed a better performance of object-level classification (an overall accuracy of $63 \%$ and Kappa coefficient of 0.559 were achieved for pixel-level classification). The performance of classes using pixel-based classification varied 45 $\%$ for Alnus incana to $80 \%$ for Picea abies. In general, Spectral-spatial complexity reduction using MNF transformation and object-level classification yielded a statistically satisfactory results.
\end{abstract}

Keywords: minimum noise fraction (MNF) transformation; object-based image analysis (OBIA); APEX hyperspectral imagery; Random forest (RF) classifier; multiresolution segmentation (MRS); tree species classification

\section{Introduction}

Tree species composition is an important key element for plant diversity and sustainable forest management [1]. Several studies have shown that hyperspectral imagery (also known as imaging spectrometers) providing a high-spectral resolution which can be used to effectively discriminate tree species [2-4]. However, there are two main challenges for analysis of hyperspectral imagery: a) Hughes phenomena or "a curse of dimensionality" [5], and b) a problem of spectral variability within an image unit (pixel) [6].

According to the Hughes phenomenon, high dimensionality of hyperspectral imagery poses a problem for sample-based classifications, in such a way that by increasing the number of 
hyperspectral bands, the number of sufficient training samples to maintain minimum statistical confidence for classification grows exponentially [7-9]. Additionally, a high number of bands causes information redundancy, meaning information content of one spectral bands can be fully or partly predicted from other bands [10]. Minimum noise fraction transformation (MNF) has been widely used to segregate noise and reduce dimensionality of hyperspectral imagery [11]. MNF is a transformation-based feature extraction, based on two cascaded principal component analyses: first, it uses principal components to de-correlate noise data based on covariance matrix, and second, it reorders the new components based on decreasing signal-to-noise ratio, resulting in the ranked spectral bands from highest variance to the highest noise [12]. Applying MNF transformation before tree species classification is expected to increase the classification accuracy [13-17]. In this study, MNF transformation was accepted and used as a main framework for reducing a spectral dimensionality of an airborne hyperspectral imagery.

The second challenge refers to analysis of spectral variability per image unit (i.e., pixel). In recent years, classification of hyperspectral imagery using spatial context along with spectral information spatial-spectral gains attentions [8, 18,19]. Object-based image analysis (OBIA), or broader spatial image analysis, uses image-objects rather than image pixels to utilize additional shape, geometry- or topology-related features in image classification [20-22]. Here, the primary goal of object-level classification is to reduce the spatial complexity of an image, by merging pixels together, and forming homogeneous segments, or image-objects. The image-objects are homogeneous regions which can be formed by various segmentation techniques. One popular region growing segmentation technique is the multiresolution segmentation (MRS) [23]. The MRS algorithm merges adjacent pixels according to not only the spectral information, but also contextual information, such as shape and compactness. The adjacent pixels should fulfil a degree of fitting for a possible merging. A merging will stop, when there are no more possible merges. MRS introduces several, interdependent hierarchical levels of image objects, so that hierarchical object relationships can be built.

All being said, the main objective of this research is to assess spectral-spatial complexity reduction, using MNF transformation and object-based image analysis for an airborne hyperspectral imagery.

\section{Materials and Data}

\subsection{Study area}

The study area is located at the North-West of the city Salzburg, over a length of 8.5 kilometres

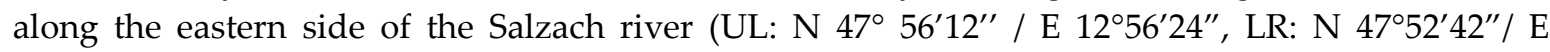
$12^{\circ} 59^{\prime} 21^{\prime \prime}$ ), Austrian-German border (Figure 1). The average altitude is 400 meters. The average annual rainfall of the area is $1200 \mathrm{~mm}$, with a maximum rainfall in summer. The forested area is a mixture of dominant and plantation trees, water bodies and wetlands, buildings and industrial areas. The plantation trees in the area comprises Picea abies (PiAb), Populus canadensis (PoCa), and Populus balsamifera (PoBa). More common native tree species found in the area are Fraxinus excelsior (FrEx), Alnus incana (AlIn) and Salix alba (SaAl), whereas, less common native tree species are Acer pseudoplatus and Quercus robur [24]. 


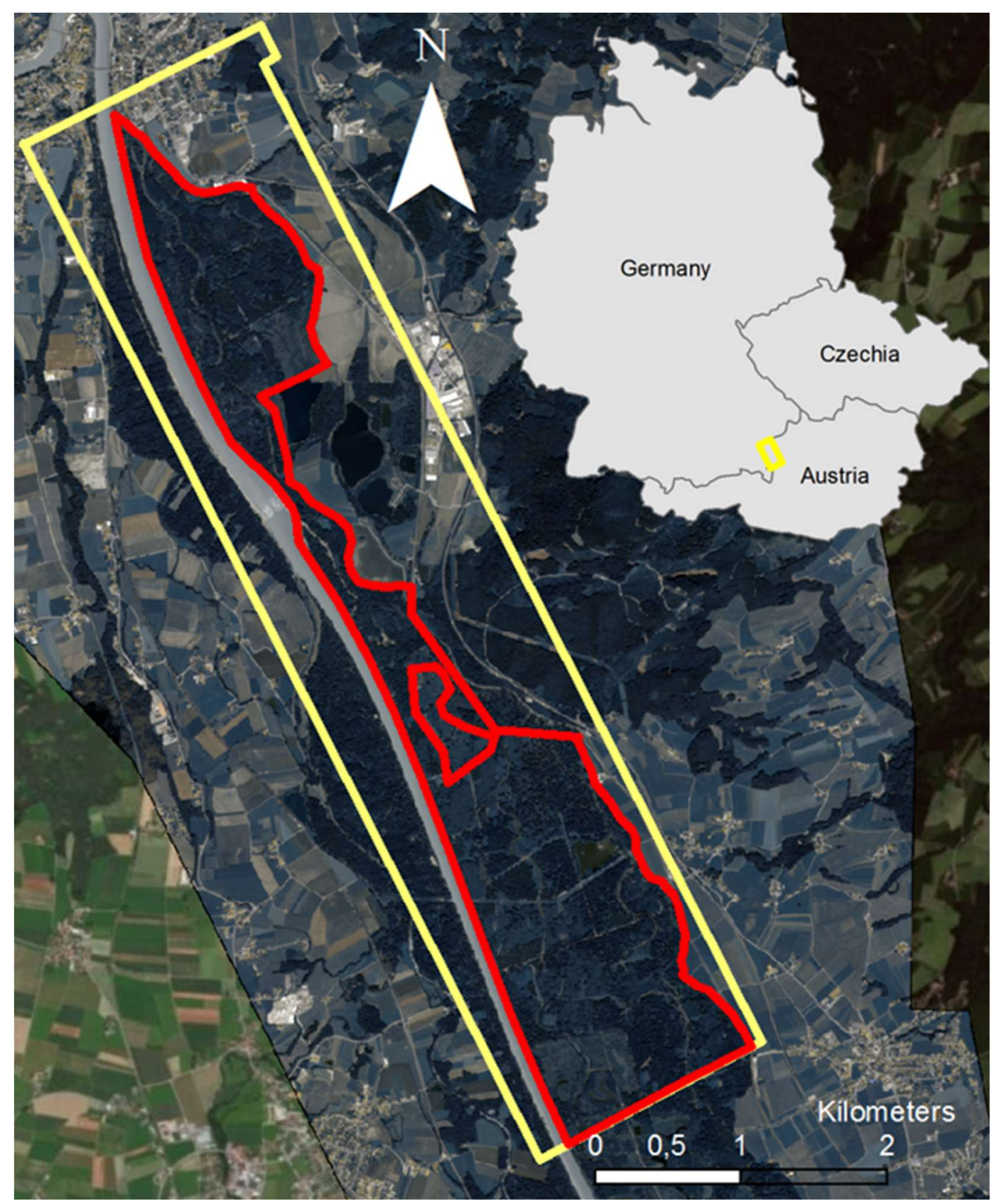

Figure 1. Salzach floodplain, located at the border of Germany and Austria. The yellow polygon indicated the airborne APEX hyperspectral imagery and the red polygon indicates the study area.

\subsection{Data}

\subsubsection{Airborne Remote Sensing Data}

For the study, an airborne ESA-APEX (airborne prism experiment) hyperspectral imagery [25] collected on 29 June 2011 was used. The APEX hyperspectral imagery had 288 number of bands (with a spectral coverage of $413.1450 \mathrm{~nm}$ to $2451.8245 \mathrm{~nm}$ ), and spatial ground resolution of $2.5 \mathrm{~m}$. The image contained two geometrically and atmospherically corrected flight lines. There were two black lines in the image, due to the presence of wires which were placed on the entry slit at the time of the image acquisition, to observe spatial shifts.

\subsubsection{Training-polygons}

Training polygons were digitized, using GPS points (which were gathered during a survey on July 2017, and Juno 7x device), visual inspection (using a pan-sharpened very-high resolution 
WorldView2 multispectral imagery with $0.5 \mathrm{~m}$ spatial resolution), and a preliminary habitat interpretation according to the EU habitat council directive [26]. It is argued that for any statisticallybased classifier (supervised classifications), the theoretical lower limit of the number of samples that must be contained in a training set is $n+1$, where $\mathrm{n}$ is the number of spectral bands [27]. However, in practice, a minimum of from $10 n$ to $100 n$ pixels were used, because the estimation of the mean vectors, and covariance matrix improves as the number of pixels in the training sets increases [27]. Accordingly, a minimum of 10 polygons per class were digitized, in such a way that either a tree crown or uniform tree stands were visible (on both very high-resolution WorldView-2 and the APEX hyperspectral imagery).

\section{Methods}

\subsection{Data Pre-processing}

\subsubsection{Removing noisy bands}

Out of the 288 bands of the APEX hyperspectral imagery 18 bands had low signal to noise ratio, due to the atmospheric major absorption and mainly by water vapor. These bands, which were characterized with a higher mean value $(>0.8)$ and less standard deviation $(<0.2)$ ranging from 1359 $\mathrm{nm}$ to $1406 \mathrm{~nm}$ and from $1813 \mathrm{~nm}$ to $1921 \mathrm{~nm}$, were excluded from the further analysis.

\subsubsection{Masking Non-Forested Areas}

The non-forested areas (such as buildings, roads, rivers and fields) were masked out from the APEX hyperspectral image by creating a non-forest vector shapefile. Excluding non-forested areas was found to be useful for applying MNF transformation, because it may reduce spectral influence of non-forested classes. Therefore, the data-transformation would be more representative of withinclass heterogeneity (forest trees) rather than between forest and non-forest classes [11].

\subsubsection{Dimensionality Reduction Minimum Noise Fraction (MNF) Transformation}

A forward Minimum noise fraction (MNF) transformation was applied on APEX hyperspectral imagery. The first $25 \mathrm{MNF}$-derived components showed the value more than 1, meaning they were carrying the largest part of information. Among the 25 MNF-derived components, 9 components were chosen by a visual inspection, where tree crowns were mostly visible (Table 1).

Table 1. MNF components were selected, based on a) the Eigenvalues, where the values were greater than 1 , and b) visual inspection, where the tree crowns were visible.

\begin{tabular}{llll}
\hline MNF-Comp. $^{1}$ & Eigenvalue & MNF-Comp. & Eigenvalue \\
\hline 1 & 170.56 & 15 & 1.518 \\
7 & 3.468 & 17 & 1.349 \\
9 & 2.418 & 18 & 1.175 \\
13 & 1.740 & 20 & 1.047 \\
14 & 1.649 & & \\
\hline \multicolumn{3}{c}{ Components. }
\end{tabular}

\subsection{Spectral Variability among Tree Species}

The mean spectral signature of six tree species created using the original airborne APEX image, and MNF-derived components (Figure 2). 


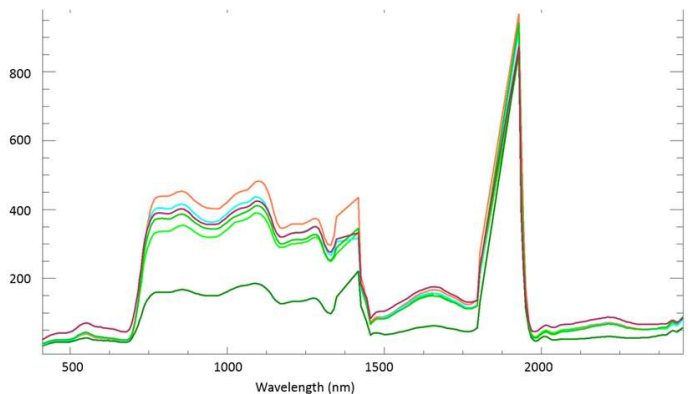

(a)

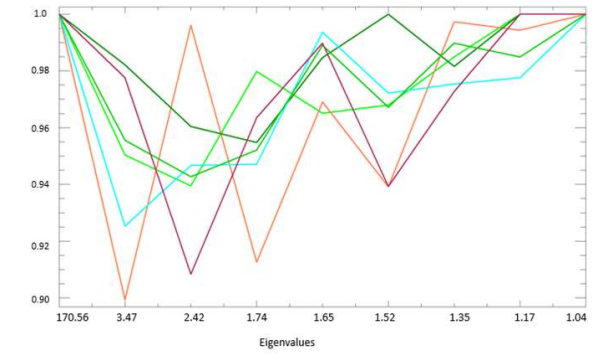

(b)
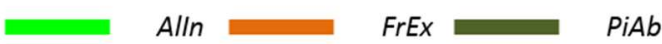

PiAb

$\mathrm{PoBa}$

PoCa

SaAl

Figure 2. This figure shows a) Spectral variability between tree species (i.e., deciduous and coniferous) were clearly disguisable in near-infrared and short-infrared portion of the spectrum. However, among tree species (except for SaAl with a very high reflectance), the difference between the spectra signature were subtle. Figure b) shows spectral variability for the same tree species using the reduced-spectral dimensionality APEX image. A correlation between bands, and difference among spectral signature were more evident. For example, in the second MNF-derived component (Eigenvalue of 3.47) nearly all the tree species showed observable differences.

\subsection{Tree Species Classification}

The random forest (RF) algorithm has been argued to be an appropriate statistical tool for classification of ecology and biodiversity related features [28]. RF was also recommended for handling more complex data such as hyperspectral imagery [29-32]. The RF algorithm is a collective classifier that uses a set of binary classification and regression trees (CARTs) to make a prediction [33]. There are two main parameters to create the forest-trees in RF algorithm, namely: number of features $\left(\mathrm{M}_{\mathrm{try}}\right)$, and number of trees $\left(\mathrm{N}_{\text {tree }}\right)$. According to literature, the classification accuracy is less sensitive to $N_{\text {tree }}$ than to $M_{\text {try }}$ parameters [11]. The value of Ntree was recommended to be set to 500, mainly because the errors stabilize before this tree-numbers was achieved, and $\mathrm{M}_{\text {try }}$ parameter was recommended to be set to the square root of the number of variables, mainly due to computational purpose [34]; therefore, in this study, all the $9 \mathrm{MNF}$-derived components were used to train the RF model. To compare the performance of the RF algorithm, the classification was of MNF-derived components were done on two levels: a) pixel-level, and b) object-level.

\subsubsection{Tree Species Classification using RF on the Pixel-Level}

Tree species classification using RF algorithm on the pixel-level was carried out using EnMAP toolbox [35]. For the training of RF model on a pixel-level, $\mathrm{N}_{\text {tree }}$ and $\mathrm{M}_{\text {try }}$ parameters were set to 500 and 9 respectively. A Gini coefficient was used for an impurity function. The produced model was then used for classification of nine MNF-derived components.

\subsubsection{Tree Species Classification using RF on the Object-Level}

Using the multiresolution segmentation (MRS) algorithm implemented in eCognition software was used for creating image-objects, the following five steps were performed:

1. Import training-polygons into the OBIA framework. The so-called test-training-area (TTA) mask was created and used later for the classification.

2. Apply MRS to all the 9 MNF-derived components. The minimum possible scale parameter of 2 for the SP was used. The homogeneity criteria of shape and compactness were set as defaults (shape criteria: 0.1, compactness: 0.5 ).

3. Overlaying and intersecting the TTA mask with the MRS results allowing a maximum overlap of $70 \%$ between TTA mask and segments. The value of $70 \%$ was chosen after different settings to get the best results. The resulting samples on MNF 
derived components were inspected visually, to avoid false positive samples. Table 2 showed the number of samples used for the classification.

Table 2. Detailed information of training-samples polygons and their area coverage.

\begin{tabular}{ccc}
\hline Tree species & $\begin{array}{c}\text { Nr. Of } \\
\text { training- } \\
\text { polygons }\end{array}$ & Area $\left.\mathbf{( s q}^{\mathbf{2}}\right)$ \\
\hline ALIn & 10 & 838 \\
$F r E x$ & 12 & 452 \\
$P i A b$ & 12 & 785 \\
$P o C a$ & 15 & 3133 \\
$P o B a$ & 12 & 849 \\
$S a A l$ & 13 & 839 \\
\hline
\end{tabular}

4. Train the RF algorithm and apply it. The mean value of each image object was calculated and used for training of RF classifier. Mtry and Ntree in the eCognition software is corresponded to a depth and a maximum tree number.

5. Export the classified objects and attribute data as a vector layer, to be used in GIS environment.

\section{Results}

\subsection{Overall Classification Results}

Examples of the classification results for both pixel- and object-based levels were presented in the Table 3.

Table 3. An example of (more or less) homogenous forest patches (one-species dominated) and the classification results of six-major tree species, on the pixel-level and on the object-level, using MNF-derived components and RF algorithm.

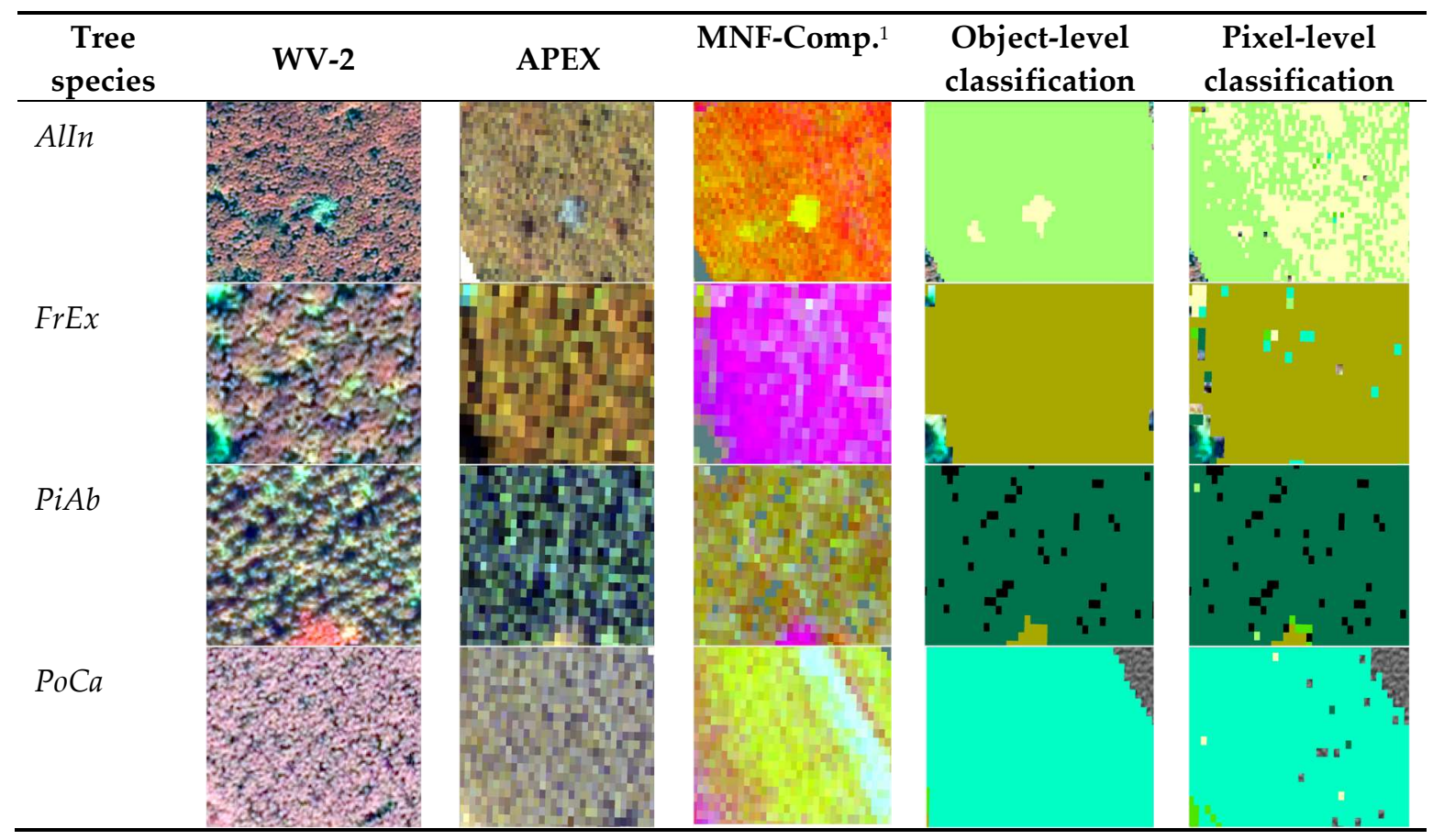




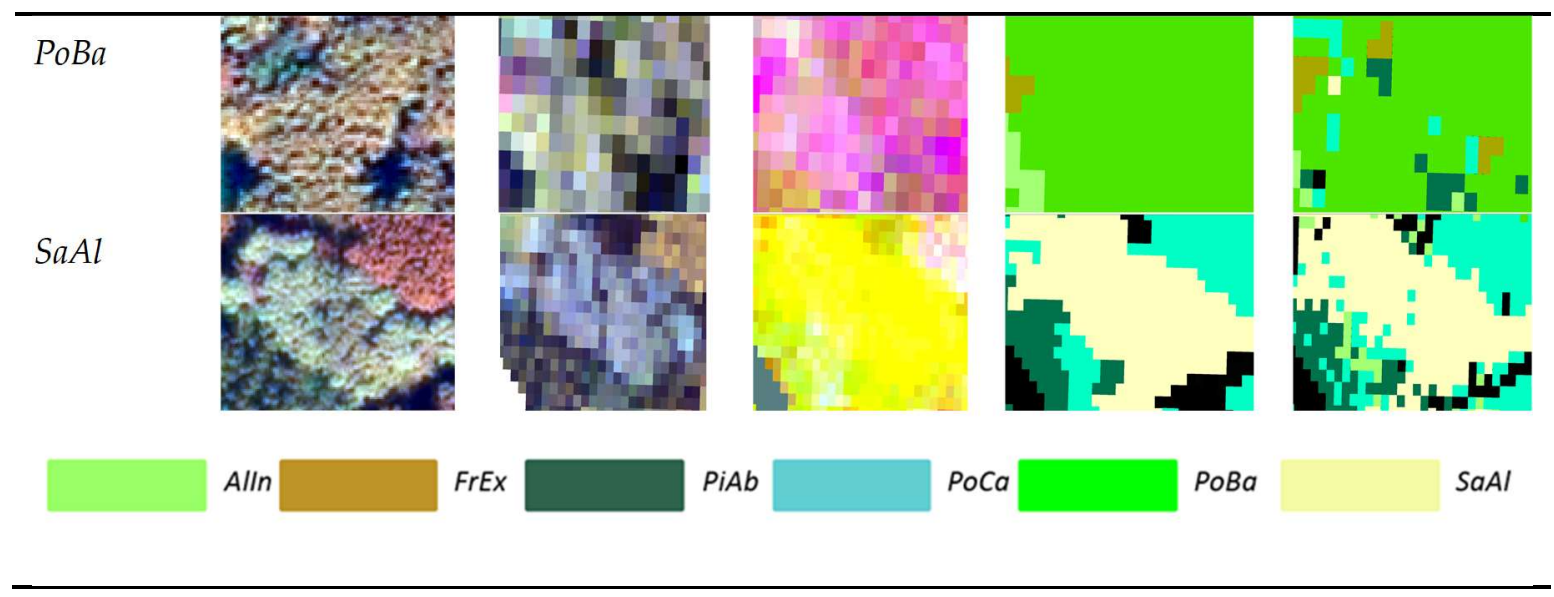

A visual inspection was performed to check consistency, by comparing the results with the pansharpened very high-resolution WorldView-2) imagery. In addition, the classification accuracy was assessed quantitatively using a producer-, a user-, and an overall-accuracy derived from a confusion matrix and a kappa coefficient [36-38]. The validation samples were created using random sampling, with minimum number of 50 samples per tree-class [39]. The results of overall accuracy and the Kappa coefficient are shown in the Table 4.

Table 4. The overall accuracy and kappa coefficient of both classification approaches.

\begin{tabular}{ccc}
\hline Classification & $\begin{array}{c}\text { Overall } \\
\text { accuracy }\end{array}$ & $\begin{array}{c}\text { Kappa } \\
\text { coefficient }\end{array}$ \\
\hline Pixel-level & $63 \%$ & 0.559 \\
Object-level & $85 \%$ & 0.805 \\
\hline
\end{tabular}

The overall accuracy of $85 \%$ and the kappa coefficient of 0.805 clearly indicate a better performance of object-level classification compare to the overall accuracy of $63 \%$ and the kappa coefficient of 0.559 for the pixel-level classification. To compare the results of the classification on two levels (i.e., object-level versus pixel-level), a McNemar's test was used. The McNemar's test is recommended for testing a statistical significant of two classifications with the same validationsamples. The assumption is that, using the same samples for validation of both classifications, an assumption of samples-independency was not fulfilled [40]. The McNemar's test is based upon confusion matrices that are 2 by 2 in dimensions, and is based upon the standardized normal test statistics:

$$
Z=\left(f_{12}-f_{21}\right) / \sqrt{ }\left(f_{12}+f_{21}\right)
$$

For any two arbitrary classifiers ( $\mathrm{C} 1$ and $\mathrm{C} 2)$, if $\mathrm{f}_{12}$ is a number of cases correctly classified by $\mathrm{C} 2$ but not $\mathrm{C} 1$, and $\mathrm{f}_{21}$ indicates the number of cases correctly classified by $\mathrm{C} 1$ but not $\mathrm{C} 2$, the null hypothesis indicates that both of the classifiers, $\mathrm{C} 1$ and $\mathrm{C} 2$ should have the same error rate, i.e., $\mathrm{f}_{12}=\mathrm{f}_{21}$ [11]. To test the hypothesis, a chi-square $\left(\chi^{2}\right)$ distribution was used, with 1 degree of freedom:

$$
\chi^{2}=\left(\left|f_{12}-f_{21}\right|-1\right) 2 /\left(f_{12}+f_{21}\right)
$$

The difference between pixel-level and object-level classification was statistically significant due to the Two-tailed P-value less than 0.0001 . 


\subsection{RF Classification Performance for each Class}

The performance of object-level and pixel-level classification were compared according to producer's and user's accuracies (Tables 5 and 6).

Table 5. Confusion matrix for RF classification using pixel-level classification. The top row of classes represents the reference classes, and the left column represents classified tree species.

\begin{tabular}{|c|c|c|c|c|c|c|c|c|}
\hline & $F r E x$ & AlIn & PoBa & PoCa & $P i A b$ & $S a A l$ & Sum & $\begin{array}{c}\text { User's } \\
\text { accuracy } \\
(\%)\end{array}$ \\
\hline$F r E x$ & 27 & 3 & 6 & 1 & 0 & 1 & 38 & 71 \\
\hline AlIn & 5 & 24 & 4 & 3 & 2 & 6 & 44 & 55 \\
\hline$P o B a$ & 12 & 3 & 33 & 7 & 3 & 0 & 58 & 57 \\
\hline PoCa & 1 & 2 & 4 & 38 & 1 & 8 & 54 & 70 \\
\hline$P i A b$ & 5 & 1 & 1 & 4 & 40 & 0 & 51 & 78 \\
\hline$S a A l$ & 2 & 17 & 0 & 1 & 2 & 38 & 60 & 63 \\
\hline Sum & 4 & 4 & 0 & 1 & 2 & 2 & 13 & \\
\hline $\begin{array}{c}\text { Producer's } \\
\text { accuracy (\%) }\end{array}$ & 56 & 54 & 48 & 55 & 50 & 55 & & \\
\hline
\end{tabular}

Table 6. Confusion matrix for RF classification using object-level classification. The top row of classes represents the reference classes, and the left column represents classified tree species.

\begin{tabular}{|c|c|c|c|c|c|c|c|c|}
\hline & FrEx & AlIn & $P o B a$ & PoCa & $P i A b$ & $S a A l$ & Sum & $\begin{array}{c}\text { User's } \\
\text { accuracy } \\
(\%) \\
\end{array}$ \\
\hline$F r E x$ & 45 & 4 & 1 & 2 & 0 & 0 & 52 & 87 \\
\hline AlIn & 3 & 43 & 3 & 4 & 3 & 0 & 56 & 77 \\
\hline$P o B a$ & 3 & 2 & 42 & 0 & 0 & 1 & 48 & 88 \\
\hline PoCa & 1 & 2 & 1 & 44 & 2 & 3 & 53 & 83 \\
\hline$P i A b$ & 2 & 2 & 1 & 4 & 45 & 1 & 55 & 82 \\
\hline$S a A l$ & 2 & 1 & 0 & 1 & 0 & 50 & 54 & 93 \\
\hline Sum & 56 & 54 & 48 & 55 & 50 & 55 & & \\
\hline $\begin{array}{c}\text { Producer's } \\
\text { accuracy (\%) }\end{array}$ & 80 & 80 & 88 & 80 & 90 & 91 & & \\
\hline
\end{tabular}

A comparison of the results indicated that the object-level classification was visually friendlier than the pixel-level classification. Additionally, according to the statistical measures, the object-level classification had a better performance in a comparison with the pixel-level classification (Table 4). According to the Producer's accuracy, the performance of classes, in the object-level classification, was ranging from from 80\% (for FrEx, AlIn, PoBa, and PoCa) to 90\% (for SaAl and PiAb). The User's accuracy, for the object-level classification, was ranging from 77\% (AlIn) to 93\% (SaAl). According to the Producer's accuracy, for the pixel-level classification, the worst results belonged to two-broad leaves species FrEx (48\%) and AlIn (45\%) and the highest performance belonged to PiAb (80\%); all three other species (PoBa, PoCa, and SaAl) showed 69\% for Producer's accuracy. The performance of 
the classes according to User's accuracy for pixel-level classification, varied from $55 \%$ for AlIn and $57 \%$ for PoBa, to $63 \%$ for SaAl. The performance for PoCa, FrEx, and PiAb were 70\%, 71\%, and 78\%.

\section{Discussion and Conclusions}

The results of the spectral-spatial dimensionality reduction of airborne APEX hyperspectral imagery, using MNF transformation and object-level classification showed a better performance in a comparison to classification of the only spectrally-reduced APEX hyperspectral imagery. Still, both classification results (i.e., object- and pixel-levels) have misclassifications, which might be caused by variation of spectral signature due to age, health and bidirectional reflectance of tree species [41-44]. Additionally, there were several other tree-species presented in the area, which due to difficulties of sample design, have not been taken into account, such as Quercus robur, and Acer pseudoplatus.

The accuracy of any supervised classification is influenced by designing samples (training and validation), and sample size. A "wall to wall" comparison of a classification result with reference data would conquest the whole purpose of performing remote-sensing based classification [27]. As a substitute, random or stratified sampling are preferable, although each of them has its limitations. Earlier, in sampling design, it was mentioned that the recommendation of the minimum 10 trainingsamples per class [39] was used. For designing validation samples, we followed again the guideline given by the same authors [39], who were suggesting a minimum of 50 validation-samples for each map-class and for maps of less than 1 million acres in size and fewer than 12 classes, for addressing accuracy using confusion matrix.

A use of bootstrap sampling prior to classification might improve classification accuracy $[45,46]$. The RF algorithm forms many classification trees, and each tree is trained based on a bootstraps sample for the training data [29]. The RF algorithm does the bootstraps internally, therefore, we didn't consider applying bootstraps separately on the training-polygons. In addition to the mentioned factors, a visual comparison of the results showed that most misclassifications were occurred in shadowing areas and where the two artificial black-lines where existed. Handling shadowing-, and noise-areas (or other abnormalities in an image) prior to the classification might have been optimizing the classification results. Moreover, the pixel-level classification results could be enhanced by postprocessing techniques, however, to avoid missing tree-classes, no post-processing strategy was applied in this study.

Lastly, the use of OBIA in this study was limited to creating homogenous objects, and creation of object-level as a base for further classification. Nevertheless, incorporating spatial information (including area, length, width, and direction), morphological criteria (such as shape parameters and texture), and spatial context may increase the tree-species classification accuracy. One of the main challenges regarding image segmentation is the determination of SP, which in return may resulted in over- or under-segmentation of the image. It was reported, that from the classification perspective, each over-segmented object (meaning one semantic object is divided into multiple smaller objects), still has a potential to be classified into true class, whereas for under-segmentation object (meaning different semantic objects merging into one large image object), is impossible to classify into their true class [47]. Therefore, in this study, the lowest possible SP was used for MRS.

As a final conclusion, the assessment of reducing the spectral dimensionality coupled with reducing spatial complexities through OBIA methods showed a satisfactory tree species classification results. MNF transformation was used for spectral dimension reduction, however, in literature, Fisher's linear discriminate analysis (FLDA) has been also applied successfully for dimensionality reduction of hyperspectral imagery [7,48]. A performance of FLDA for dimensionality reduction in a comparison with MNF transformation could be assessed in a future work.

\section{Author Contributions:}

Zahra Dabiri was responsible for the conceptualization and methodological development, overall data analysis, and writing of the manuscript. Stefan Lang provided remote sensing imagery, provided overall supervision, as 
well as detailed scientific support for the development of the research, and reviewed the manuscript. Field-trip was done by both authors.

Acknowledgments: The research leading to these results has been supported by the European Community's Seventh Framework Programme (FP7/2007-2013) under grant agreement No. 263479.(MS.MONINA) and the Austrian Science Fund (FWF) through the Doctoral College GIScience (DK W1237-N23).

Conflicts of Interest: The authors declare no conflict of interest.

\section{References}

1. Barbier, S.; Frédéric, G.; Philippe, B. Influence of tree species on understory vegetation diversity and mechanisms involved - a critical review for temperate and boreal forests, Forest ecology and management, 2008, 254, 1-15, doi: 10.1016/j.foreco.2007.09.038.

2. Clark, M. L.; Roberts. D. A; Clark, D. B. Hyperspectral discrimination of tropical rain forest tree species at leaf to crown scales, Remote Sens. Environ. 2005, 96, 375-398, doi: 10.1016/j.rse.2005.03.009.

3. Dalponte, M.; Orka, H. O; Gobakken, T.; Gianelle, D; Næsset, N. Tree species classification in boreal forests with hyperspectral data, IEEE Transaction on Geoscience and Remote sensing, 2013, 51, 2632-2645, doi:10.1109/TGRS.2012.2216272

4. Clark, M. L. Identification of Canopy Species in Tropical Forests Using Hyperspectral Data. In Hyperspectral Remote Sensing of Vegetation, Thenkabail, P.S.; Lyon, J. G; Huete, A., Eds.; CRC Press, Boca Raton, FL, USA, 2011, Chapter 18, 423-445 (Google Scholar).

5. Hughes, G. On the mean accuracy of statistical pattern recognizers, Transaction on information theory, 1968, 14, 55-63, doi:10.1109/TIT.1968.1054102.

6. Plaza, A.; Martinez, P.; Perez, R; Plaza, J. A new approach to mixed pixel classification of hyperspectral imagery based on extended morphological profiles, Pattern Recognition, 2004, 37 1097-1116, doi: 10.1016/j.patcog.2004.01.006

7. Melgani, F.; Bruzzone, L. Classification of hyperspectral remote sensing images with support vector machines, IEEE Transaction on Geoscience and Remote Sensing, 2004, 42, 1778-1790, doi: 10.1109/TGRS.2004.831865.

8. Plaza, A.; Benediktsson, J. A.; Boardman, J. W.; Brazile, J.; Bruzzone, L; Camps-Valls, G.; Chanussot, J.; Fauvel, M.; Gamba, P.; Gualtieri, A. Recent advances in techniques for hyperspectral image processing, Remote Sens. Environ. 2009,13, 110-122, doi: 10.1016/j.rse.2007.07.028.

9. Foody, G. M.; Mathur, A. Toward intelligent training of supervised image classifications: directing training data acquisition for SVM classification, Remote Sens. Environ. 2004, 93, 107-117, doi: 10.1016/j.rse.2004.06.017

10. Thenkabail, A.; Lyon, P. S.; Huete, J. G.; Bajwa, S. G.; Kulkarni, S. S. Hyperspectral Data Mining. In Hyperspectral Remote Sensing of Vegetation, Thenkabail, P.S.; Lyon, J. G; Huete, A., Eds.; CRC Press, Boca Raton, FL, USA, 2011, Chapter 4, 93-120 (Google Scholar).

11. Ghosh, A.; Fassnacht, F. E.; Joshi, P. K.; Koch; B. A framework for mapping tree species combining hyperspectral and LiDAR data: Role of selected classifiers and sensor across three spatial scales; International Journal of Applied Earth Observation and Geoinformation , 2014, 26, 49-63, doi: 10.1016/j.jag.2013.05.017.

12. Van der Meer, F.D.; De Jong, S. M. Imaging spectrometry: basic principles and prospective applications. In imaging Spectrometry: Basic Principles and Perspective Applications, Van der Meer, F.D.; De Jong, S. M, EDS.; Springer Science \& Business Media, Dordrecht, The Netherland, 2011, Vol. 4.

13. Underwood, E.; Ustin, S.; DiPietro, D. Mapping nonnative plants using hyperspectral imagery, Remote Sens. Environ. 2003, 86, 150-161, doi: 10.1016/S0034-4257(03)00096-8.

14. Zhang, D.; Le, Y.Support vector machine based classification for hyperspectral remote sensing images after minimum noise fraction rotation transformation. In the Internet Computing \& Information Services (ICICIS), Hong Kong, China, 2011, IEEE, doi: 10.1109/ICICIS.2011.39.

15. Zhang, C.; Xie, Z. Combining object-based texture measures with a neural network for vegetation mapping in the Everglades from hyperspectral imagery, Remote Sens. Environ. 2012, 124, 10-20, doi.org/10.1016/j.rse.2012.05.015. 
16. Onojeghuo, A. O.; Blackburn, G. A. Optimising the use of hyperspectral and LiDAR data for mapping reedbed habitats, Remote Sens. Environ. 2011, 115, 2025-2034, doi.org/10.1016/j.rse.2011.04.004.

17. Belluco, E.; Camuffo, M.; Ferrari, S.; Modenese, L.; Silvestri, S.; Marani, A.; Marani, M. Mapping salt-marsh vegetation by multispectral and hyperspectral remote sensing, Remote Sens. Environ. 2006, 105, 54-67, doi.org/10.1016/j.rse.2006.06.006.

18. Fauvel, M.; Tarabalka, Y.; Benediktsson, J. A.; Chanussot, J.; Tilton, J. C. Advances in spectral-spatial classification of hyperspectral images. In proceedings of the IEEE, 2013, 101, 652-675, 10.1109/JPROC.2012.2197589.

19. Ghamisi, P.; Mura, M. D.; Benediktsson, J. A. A survey on spectral-spatial classification techniques based on attribute profiles, IEEE Transaction on Geoscience and Remote Sensing, 2015, 53, 2335-2353, doi: 10.1109/TGRS.2014.2358934.

20. Blaschke, T. Object based image analysis for remote sensing, ISPRS J. Photogramm. Remote Sens. 2010, 65, pp. 2-16, doi:10.1016/j.isprsjprs.2009.06.004

21. Blaschke, T.; Hay, G.J.; Kelly, M.; Lang, S.; Hofmann, P.; Addink, E.; Feitosa, R. Q.; Van der Meer, F.; Van der Werff, H.; Van Coillie, F.; Tiede, D. Geographic object-based image analysis-towards a new paradigm, ISPRS J. Photogramm Remote Sens. 2014, 87,180-191, doi: 10.1016/j.isprsjprs.2013.09.014.

22. Lang, S. Object-based image analysis for remote sensing applications: modeling reality - dealing with complexity. In Object based Image Analysis- Spatial concepts for knowledge-driven remote sensing applications, Blaschke, T.; Lang, S.; Hay, G., Eds.; Springer: Berlin, Germany, 2008, 3-28, ISBN 978-3-54077057-2.

23. Baatz, M.; Schäpe, A. Multiresolution segmentation-an optimization approach for high quality multi-scale image segmentation. In Angewandte Geographische Informationsverarbeitung, Strobl, J., Blaswchke, T., Griesebner, G., Eds.; Wischmann-Verlag: Heidelberg, Germany, 2000, Volume 12, 12-23.

24. Strasser, T.; Lang, S. Object-based class modelling for multi-scale riparian forest habitat mapping, Int. J. Appl. Earth Obs. Geoinf. 2015, 37, 29-37, doi:10.1016/j.jag.2014.10.002.

25. Itten, K. I.; Dell'Endice, F.; Hueni, A.; Kneubühler, M.; Schläpfer, D.; Odermatt, D.; Seidel, F.; Huber, S.; Schopfer, J.; Kellenberger, T; Buehler, Y.; D’Odorico, P.; Nieke, J.; Alberti, E.; Meuleman, K. APEX-The hyperspectral ESA airborne prism experiment, Sensors, 2008, 8, 6235-6259, doi: 10.3390/s8106235.

26. HabDir, Council Directive 92/43/EEC, https://eur-lex.europa.eu/legalcontent/EN/TXT/?uri=celex\%3A31992L0043, (accessed on 10.06.2018).

27. Lillesand, T.; Kiefer, R. W.; Chipman. J. Remote sensing and image interpretation, John Wiley \& Sons (Asia), Singapor, 2004, Chapter 7, ISBN: 81-265-1335-7.

28. Cutler, D. R.; Edwards, T. C.; Beard, k. H.; Cutler, A.; Hess, K. T.; Gibson, J.; Lawler, J. J. Random forests for classification in ecology, Ecology, 2007, 88, 2783-2792, doi: 10.1890/07-0539.1

29. Gislason, P. O.; Benediktsson, J. A.; Sveinsson, J. R. Random forests for land cover classification. Pattern Recognition Letters, 2006, 27, 294-300, doi: 10.1016/j.patrec.2005.08.011.

30. Bosch, A.; Zisserman, A.; Munoz, X. Image classification using random forests and ferns. In the Computer Vision (ICCV), Proceedings of the IEEE 11th International Conference, Rio de Janerio, Brazil, 2007, doi: 10.1109/ICCV.2007.4409066.

31. Chan, J.C.W.; Paelinckx, D. Evaluation of Random Forest and Adaboost tree-based ensemble classification and spectral band selection for ecotope mapping using airborne hyperspectral imagery, Remote Sens Environ. 2008, 112, 2999-3011, doi: doi.org/10.1016/j.rse.2008.02.011.

32. Rodriguez-Galiano, V. F.; Ghimire, B.; Rogan, J.; Chica-Olmo, M.;Rigol-Sanchez, J. P. An assessment of the effectiveness of a random forest classifier for land-cover classification., ISPRS. J. Photogramm. Remote Sens. 2012, 67, 93-104, doi: 10.1016/j.isprsjprs.2011.11.002

33. Breiman, L. Random forests, Machine learning, 2001, Volume 45, 5-32, doi: 10.1023/A:1010933404324.

34. Belgiu, M.; Drăguț, L. Random forest in remote sensing: A review of applications and future directions, ISPRS. J. Photogramm. Remote Sens. 2016, 114, 24-31, doi: 10.1016/j.isprsjprs.2016.01.011.

35. Van der Linden, S.; Rabe, A.; Held, M.; Jakimow, B.; Leitão, P. J.; Okujeni, A.; Schwieder, M.; Suess, S.; Hostert, P. The EnMAP-Box-A toolbox and application programming interface for EnMAP data processing, Remote Sens. 2015, 7, 11249-11266, doi: 10.3390/rs70911249.

36. Congalton, R. G. Remote Sensing and Geographic Information System Data Integration: Error Sources and Research Issues, PE \& RS, 1991, 57, 677-687. 
37. Congalton, R.G. Accuracy assessment of remotely sensed data: Future needs and directions. Land Information from Space-based Systems, In Proceedings of Pecora 12, ASPRS, Bethesda, USA, 1994, 383-388.

38. Smits, P.C.; Dellepiane, S.G.; Schowengerdt, R. A. Quality assessment of image classification algorithms for land-cover mapping: a review and a proposal for a cost-based approach, Int. J. Remote Sens. 1999, 20, 1461-1486, doi: 10.1080/014311699212560.

39. Congalton, R. G.; Green, K. Assessing the accuracy of remotely sensed data: principles and practices, Congaltion, R.C.; Green, K. Eds, CRC press, Boca Raton, USA, 2008, ISBN: 978-1-4200-5512-2.

40. Foody, G. M. Thematic map comparison, Photogrammetric Engineering \& Remote Sensing, 2004, 70, 627633, doi: 10.14358/PERS.70.5.627.

41. Leckie, D. G.; Tinis, S.; Nelson, T.; Burnett, C.; Gougeon, F. A.; Cloney, E.D.; Paradine, D. Issues in species classification of trees in old growth conifer stands, Canadian Journal of Remote Sensing, 2005, 31, 175-190, doi: 10.5589/m05-004.

42. Ballanti, L.; Blesius, L.; Hines, E.; Kruse, B. Tree species classification using hyperspectral imagery: a comparison of two classifiers, Remote Sens. 2016, 8, 445, doi: 10.3390/rs8060445.

43. Buddenbaum, H.; Hill, J. Classification of coniferous tree species and age classes using hyperspectral data and geostatistical methods, Int. J. Remote Sens. 2005, 26, 5453-5465, doi: 10.1080/01431160500285076.

44. Voss, M.; Sugumaran, R. Seasonal effect on tree species classification in an urban environment using hyperspectral data, LiDAR, and an object-oriented approach, Sensors, 2008, 8, 3020-3036, doi: 10.3390/s8053020.

45. Brenning, A. Spatial cross-validation and bootstrap for the assessment of prediction rules in remote sensing: The $\mathrm{R}$ package sperrorest. In Processing of the IGARSS Geoscience and Remote Sensing Symposium, Munchen, Germany, 2012.

46. Tuia, D.; Ratle, F.; Pacifici, F.; Kanevski, M. F.; Emery, W. J. Active learning methods for remote sensing image classification, IEEE Transactions on Geoscience and Remote Sensing, 2009, 47, 2218-2232, doi: 10.1109/TGRS.2008.2010404.

47. Liu, D.; Xia, F. Assessing object-based classification: advantages and limitations, Remote Sensing Letters, 2010, 1, 187-194, doi: doi.org/10.1080/01431161003743173. 